PART I

VENUS 


\title{
THE CHEMICAL COMPOSITION OF THE ATMOSPHERE OF VENUS
}

\author{
A. P. VINOGRADOV, YU. A. SURKOV, B. M. ANDREICHIKOV, \\ O. M. KALINKINA, and I. M. GRECHISCHEVA \\ Vernadskii Institute of Geochemistry, Soviet Academy of Sciences, Moscow, U.S.S.R.
}

\begin{abstract}
The chemical composition sensors on the Venera-4, -5, and -6 spacecraft are described. The mixing ratio by volume of carbon dioxide is determined to be $97(+3,-4) \%$; nitrogen, less than $2 \%$, oxygen, less than about $0.1 \%$; and water vapor at the $25{ }^{\circ} \mathrm{C}$ temperature level, on the order of $10 \mathrm{mg} / \mathrm{l}$, an amount large enough to imply that the clouds of Venus are composed of condensed water.
\end{abstract}

\section{Introduction}

In two earlier papers, $[1,2]$ we had an opportunity to present briefly the measurements of the composition of the Venus atmosphere on the basis of data acquired with the interplanetary station* Venera-4. In this paper we present data, partly already known [3], obtained with the Venera-5 and Venera-6 spacecraft, and derive the chemical composition of the atmosphere on the basis of all the measurements available.

Until the launch of Venera-4, we had remarkably contradictory information on the chemical composition of the atmosphere of Venus. This information, as is known, was obtained from either spectral observations from the earth, or by means of various computations, comparisons, and the construction of models of the Venus atmosphere [4].

Data on the constituents $\mathrm{CO}_{2}, \mathrm{H}_{2} \mathrm{O}, \mathrm{O}_{2}, \mathrm{~N}_{2}$, and other gases had a very wide range of values. Therefore, as we noted before, in the first experiments on Venera-4 we were restricted to making threshold determinations of these components. The results of the measurements were presented at a symposium on the atmospheres of the planets, and are found in a series of reports [5].

In the subsequent investigations with the Venera- 5 and Venera- 6 spacecraft, thanks to the data from Venera-4, we were able to make determinations of these gases with greater precision and within narrower limits than before for the concentrations of $\mathrm{CO}_{2}, \mathrm{H}_{2} \mathrm{O}, \mathrm{N}_{2}$, and $\mathrm{O}_{2}$. It was thereby determined from the data of Venera-4 that the optimum regimes for the operation of the instruments could be established in accordance with the presumed conditions on the planet.

\section{Organization of the Experiments}

The Venera-4 spacecraft was launched in June, 1967. Four months later it made the first smooth descent into the atmosphere of Venus and made investigations of the physical, chemical, and structural parameters in the atmosphere of the planet. Measurements were made during the descent of the spacecraft by parachute. Those quantities

* Translator's note: The Soviets refer to their spacecraft in this paper as 'automatic interplanetary stations', 'interplanetary stations', 'landing stations', etc. For clarity, these terms are uniformly translated herein as 'space probe' or 'spacecraft'. 
measured were temperature, pressure, and composition, over the range of 0.7 atmospheres pressure and $25^{\circ} \mathrm{C}$ to $18 \mathrm{~atm}$ pressure and $280{ }^{\circ} \mathrm{C}$. In that experiment the first direct measurements in the atmosphere of a planet were made. This allowed us to restrict considerably the circle of different suppositions about the atmosphere of Venus, its origin and evolution, and to form definite ideas [1, 2]. We had placed on Venera-4 apparatus capable of a wide range of measurements which relatively coarsely, but reliably, determined the basic parameters of the atmosphere. The wide range was necessary because of the indeterminate nature of our knowledge of physical conditions on Venus, as we have already mentioned.

In January, 1969, two more space probes were sent to Venus - Venera-5 and Venera6. The purpose of the launching of these probes was to obtain further and more detailed studies of the atmosphere of the planet. As is known, these space probes penetrated deeper into the atmosphere of Venus. Measurements were made during parachute descent, and the temperature and pressure were observed to vary from $25^{\circ} \mathrm{C}$ and $0.6 \mathrm{~atm}$ to $320^{\circ} \mathrm{C}$ and $27 \mathrm{~atm}$. Apart from the investigations of temperature, pressure, and composition, the depth of the atmosphere was determined with these two probes.

As it turned out, the measured change in temperature with height in the entire interval studied is close to the adiabatic gradient. If it is assumed that the temperature varies according to the adiabatic law down to the very surface, then at the surface level as determined by the altimeter on Venera-6, the temperature and pressure must be

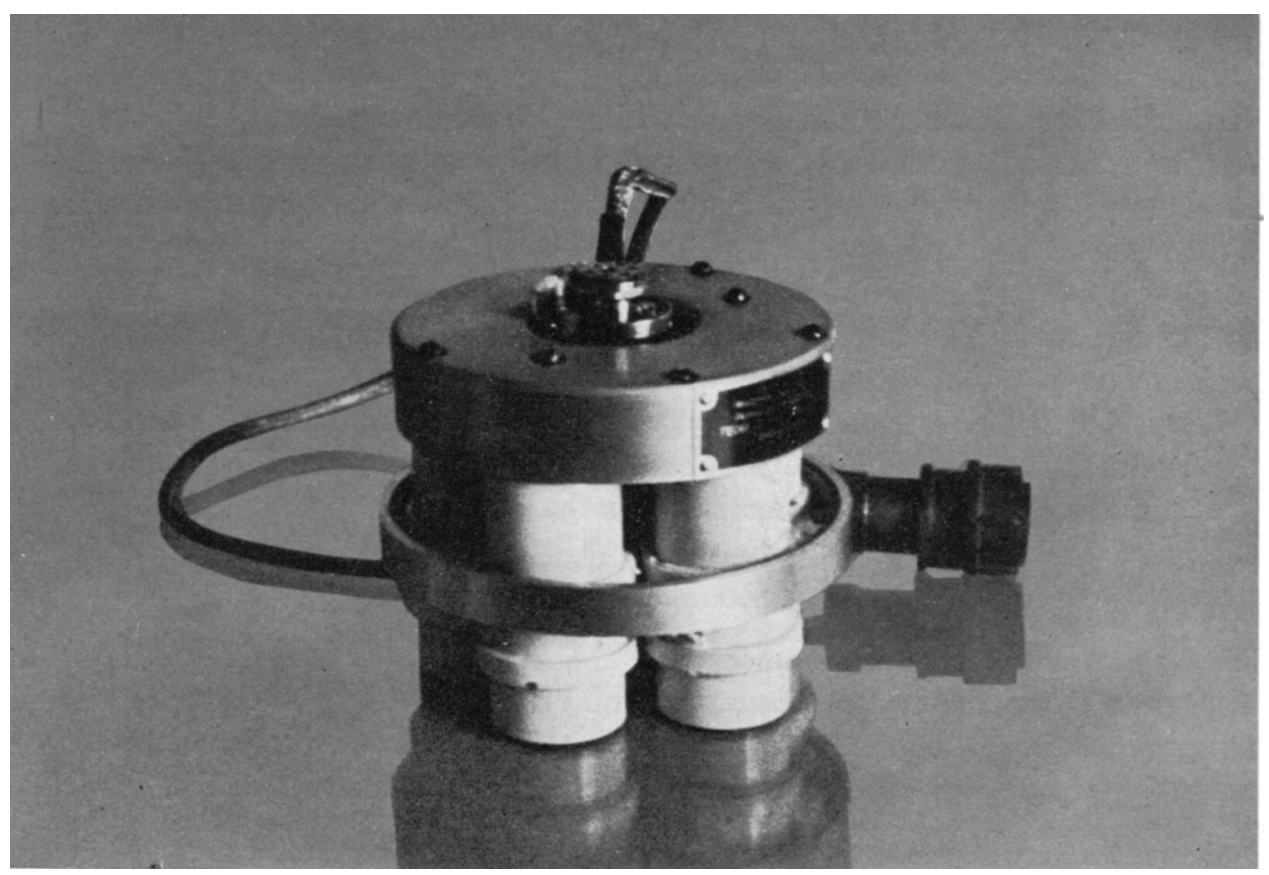

Fig. 1. General view of the gas analysis apparatus. 
$400{ }^{\circ} \mathrm{C}$ and $60 \mathrm{~atm}$, and at the surface level determined by the altimeter on Venera- 5 they must be $530{ }^{\circ} \mathrm{C}$ and 140 atm [6].

These differences in temperature and pressure in the regions of the descents of Venera-5 and Venera- 6 follow from the differences (on the order of $14 \mathrm{~km}$ ) of the level of the surface as measured by the altimeters on the probes, and reveal, it appears, the existence of large nonuniformities in the relief of the planet's surface.

At the present time we have results of measurements in two positions. Therefore, we may speak of some mean level of the surface, to which must be accorded a pressure of about $100 \mathrm{~atm}$ and temperature about $500{ }^{\circ} \mathrm{C}$. Then the levels of the surface measured by Venera- 5 and Venera- 6 would correspond to differences in altitude of $-7 \mathrm{~km}$ and $+7 \mathrm{~km}$, respectively. This mean level, fortunately, agrees with the data of radioastronomical measurements [7]. In addition, it can be refined, of course, with additional data on different regions of the surface.

On all three spacecraft we installed special gas analysis apparatus for investigations of the composition of the Venus atmosphere, and to determine the concentrations of carbon dioxide, oxygen, water, nitrogen, together with inert gases (Figure 1). On each probe were two instruments which worked in determining the altitude (pressure). The command to the two instruments came from a program-time system. The weight of each instrument was about $1 \mathrm{~kg}$, using electrical power of about $30 \mathrm{~W}$ in $10 \mathrm{sec}$ time.

In the course of the operation of all the probes in the Venus atmosphere, the concentration of carbon dioxide gas, oxygen, water, and nitrogen (together with the inert gases) was determined several times. This information was repeatedly transmitted from each instrument.

\section{Methods of Measurement, and the Results}

In the determination of the concentrations of the basic components of the atmosphere, a manometric method was mainly used. This system is remarkably simple and reliable. The sensing elements were of the pressure membrane type. Depending on the construction, these permit measurement of absolute pressure or differences in pressure between two chambers arising from absorption of the component being studied. For each component of the atmosphere, a suitable absorbent material was selected. With this method the concentration of carbon dioxide gas, nitrogen, oxygen, and water vapor was determined. In addition, in the instruments an electrolytic method was used for determination of small amounts of moisture, and a thermochemical device for small quantities of oxygen. Measurements of a few of the components of the atmosphere were repeated a second time for additional reliability. The choice of chemical absorbents was made according to the following criterion: absorbents with sufficient substance and selectivity must provide complete absorption of the gas in a fixed time interval. At the same time the speed of the reaction of absorption must not be so great as to cause noticeable changes in the composition of the gas as it was being taken into the instrument before the entrance closed.

The reagents should be stable over a sufficient range of temperature, determined 
by the technical conditions of storage and operation of the instrument, and should satisfy the indicated demands on the speed of reaction in the given range.

The reagents should not react with one another or change their characteristics upon long storage in vacuum conditions.

Gaseous products should not arise upon reaction because these would interfere with the determination of a component in a given volume.

The reagents should be able to withstand a considerable loading (this makes the use of liquids and pastes more difficult).

\section{A. DETERMINATION OF $\mathrm{CO}_{2}$ GAS}

In selecting the $\mathrm{CO}_{2}$ adsorber, the adsorptive properties of ascharite, sodium hydroxide, potassium hydroxide, and calcium hydroxide were compared. It was completely clear that acid fumes $(\mathrm{HF}, \mathrm{HCl})$ would be adsorbed by this method. However, it is known that the observed $\mathrm{HF}$ and $\mathrm{HCl}$ in the atmosphere of Venus constitute,

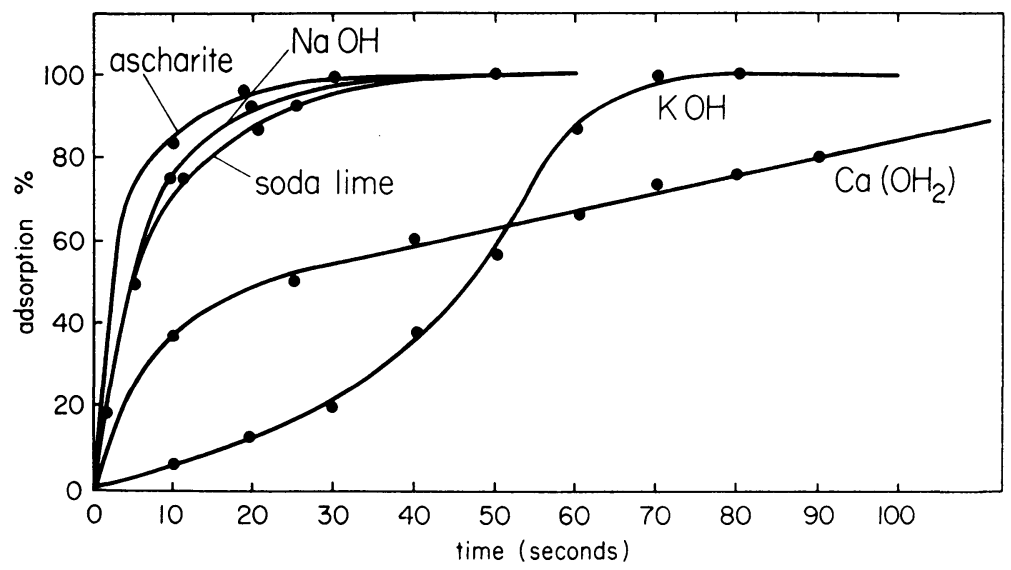

Fig. 2. Adsorption of $\mathrm{CO}_{2}$ by different adsorbent materials.

respectively, not more than $5 \times 10^{-9}$ and $6 \times 10^{-7}$ of the quantity of $\mathrm{CO}_{2}$; i.e. six to eight orders of magnitude less. Thus they can be neglected.

Figure 2 shows the dependence of adsorption of $\mathrm{CO}_{2}$ on time for various adsorbers. From the figure it can be seen that calcium hydroxide cannot be used because of the slowness of the reaction. Potassium hydroxide has a clearly expressed delayed reaction, depending on the moistness of the reagent. The adsorptive qualities of ascharite, sodium hydroxide, and soda lime are almost equal to one another. In order to use them, a minimum time of specimen intake would have been necessary. The most suitable adsorber for our use turned out to be potassium hydroxide. In order to eliminate the dependence of the speed of the reaction on the degree of desiccation, a lithium adsorber of $\mathrm{CO}_{2}$ was added to the $\mathrm{KOH}$ (about $3 \%$ ). The degree of desiccation of the reagent did not then influence the adsorption of $\mathrm{CO}_{2}$, and the adsorption was completed in $35-40 \mathrm{sec}$.

The $\mathrm{CO}_{2}$ content of the atmosphere of Venus was determined by the difference in 
pressures between compartments of a cell, in one of which was placed the $\mathrm{CO}_{2}$ adsorber. In order to avoid errors associated with the adsorption of water vapor by $\mathrm{KOH}, \mathrm{CaCl}_{2}$ was placed in the other compartment to adsorb moisture. The difference in pressure was registered using a sensor employing a membrane, as indicated above. The results of the determination of $\mathrm{CO}_{2}$ by these methods are listed in Table I.

TABLE I

Results of the determination of $\mathrm{CO}_{2}$ in the atmosphere of Venus

\begin{tabular}{lccll}
\hline Number & $\begin{array}{l}\text { Pressure } \\
\text { (atm) }\end{array}$ & $\begin{array}{l}\text { Temperature of } \\
\text { the atmospher } \\
\text { of Venus, }{ }^{\circ} \mathrm{C}\end{array}$ & $\begin{array}{l}\text { Results of } \\
\text { measurements } \\
\text { in volume } \%\end{array}$ & Spacecraft \\
\hline 1 & 0.6 & $\sim 25$ & $97 \pm 4$ & Venera-5 \\
2 & 0.7 & $\sim 25$ & $90 \pm 10$ & $\begin{array}{l}\text { Venera-4 } \\
3\end{array}$ \\
2.0 & 85 & $>56$ & Venera-6 \\
4 & 5.0 & 150 & $>60$ & Venera-5 \\
5 & 10.0 & 220 & $>30$ & Venera-6 \\
\hline
\end{tabular}

As can be seen from the table, the atmosphere of the planet contains up to 97 $(+3,-4) \%$ carbon dioxide gas. The large error in determining the carbon dioxide content by Venera- $4(90 \pm 10 \%)$ was caused by the fact that the ambient pressure at the moment of sampling was not measured and was determined by extrapolation.

In order to reduce errors in the determinations, Venera-5 and Venera- 6 were provided with means to measure the ambient pressure directly at the point where the samples for analyses were taken. This allowed us to reduce the error in determination to about $4 \%$.

Some instruments worked at pressures significantly surpassing the assumed working range of the pressure sensors, and so threshold values of the $\mathrm{CO}_{2}$ content were obtained which do not contradict the quantitative determinations. The reduction to the threshold determination of $\mathrm{CO}_{2}$ with height is connected with the increase of pressure at the same sensitivity.

Thus, by direct quantitative determinations we can accept a mean content of carbon dioxide in the atmosphere of Venus of $97(+3,-4) \%$.

\section{B. DETERMINATION OF MOLECULAR NITROGEN}

Nitrogen was determined by two methods. On Venera-4 it was determined by the difference in pressure between two compartments, in one of which all active gases, together with nitrogen, were adsorbed and in the other of which $\mathrm{CO}_{2}, \mathrm{O}_{2}$, and water vapor were adsorbed. In Venera- 5 and Venera- 6 the determination was made by means of a measurement of the pressure remaining after adsorption of the basic constituents of the atmosphere $-\mathrm{CO}_{2}, \mathrm{H}_{2} \mathrm{O}$, and $\mathrm{O}_{2}$.

Adsorption of $\mathrm{N}_{2}$ is usually accomplished by its ability to form nitrides. On investigating the metals that easily form stable nitrides, the most acceptable was found to be zirconium. It is sufficiently stable in ordinary conditions and when heated reacts well with various gases. 
The optimum temperatures for the adsorption by zirconium of the most frequently encountered gases are: for hydrogen $300{ }^{\circ} \mathrm{C}$; for oxygen $400{ }^{\circ} \mathrm{C}$; for water vapor 250-350 ${ }^{\circ} \mathrm{C}$; for nitrogen $800{ }^{\circ} \mathrm{C}$; for carbon dioxide $800{ }^{\circ} \mathrm{C}$; and for carbon monoxide $800{ }^{\circ} \mathrm{C}$.

A quartz capillary tube, inside of which a tungsten spiral was placed for heating, was used as a heater.

In order to obtain a sufficient layer of the adsorber, zirconium powder (grain size 1-5 $\mu$ ) was cemented on the quartz capillary with a $20 \%$ solution of sodium silicate. To speed and intensify the reaction an initiating mixture was used. The best results were obtained with aluminum and barium peroxide in a ratio of $2: 1$. The weight of the initiating mixture comprised about one-fifth of the weight of the zirconium adsorber.

A test showed that the presence of small quantities of $\mathrm{CO}_{2}, \mathrm{O}_{2}$, and water vapor did not affect the operation of the zirconium adsorber. As $\mathrm{O}_{2}, \mathrm{CO}_{2}$, and $\mathrm{H}_{2} \mathrm{O}$ would be adsorbed simultaneously with nitrogen, adsorbers for these gases were placed in the comparison chamber of the gas analyser. For $\mathrm{CO}_{2}$ and water, potassium hydroxide was used, and for oxygen, phosphorus.

This method was used to determine the nitrogen content only on the first spacecraft, Venera-4, since we had at first allowed for a large nitrogen content in the atmosphere of the planet. More precise data on nitrogen were completely lacking at that time. The measurements on Venera-4 showed that nitrogen is scarce $[1,2]$. Because of this, in subsequent experiments (on Venera-5 and Venera-6) the quantity of nitrogen (together with inert gases possibly present) was determined by the pressure remaining after adsorption of $\mathrm{CO}_{2}, \mathrm{O}_{2}$, and water vapor. This method was chosen because zirconium adsorbers in mixtures with $\mathrm{CO}_{2}$ present do not operate with sufficient stability.

The results of all determinations of nitrogen in the atmosphere of Venus are given in Table II.

TABLE II

Results of the determination of $\mathrm{N}_{2}$ in the atmosphere of Venus (together with possible existing inert gases)

\begin{tabular}{llcll}
\hline Number & $\begin{array}{l}\text { Pressure } \\
\text { (atm) }\end{array}$ & $\begin{array}{l}\text { Temperature of } \\
\text { the atmosphere } \\
\text { of Venus, }{ }^{\circ} \mathrm{C}\end{array}$ & $\begin{array}{l}\text { Results of } \\
\text { measurements } \\
\text { in volume } \%\end{array}$ & Spacecraft \\
\hline 1 & 0.6 & $\sim 25$ & $<3.5$ & Venera-5 \\
2 & 0.7 & $\sim 25$ & $<7^{\mathrm{a}}$ & Venera-4 \\
3 & 2 & 85 & $<2.5^{\mathrm{a}}$ & Venera-4 \\
4 & 2 & 85 & $<9.5$ & Venera-6 \\
5 & 5 & 150 & $<4$ & Venera-5 \\
6 & 5 & 150 & $<4$ & Venera-5 \\
7 & 10 & 220 & $<2.5$ & Venera-6 \\
8 & 10 & 220 & $<2$ & Venera-6 \\
\hline
\end{tabular}

${ }^{a}$ Measurement of nitrogen determined by adsorption by zirconium. The others by the pressure method. 
As can be seen from the table, nitrogen was determined eight times and all the measurements indicate its low abundance. As errors of measurement are possible, owing to incomplete adsorption, the given measurements can be taken as reliable upper limits on the nitrogen content.

Thus, we can adopt a nitrogen content in the atmosphere of Venus of not more than $2 \%$. In addition it should be noted that the quantity of inert gases, mainly argon, is evidently very small. In the atmosphere of the earth the content of argon, the most widely distributed of the inert gases, is around $1 \%$ of the amount of nitrogen in the atmosphere.

\section{DETERMINATION OF OXYGEN}

As indicated above, two methods for the determination of oxygen were used - the manometric and the thermochemical.

The simplest and best studied adsorber of oxygen is white phosphorus. The

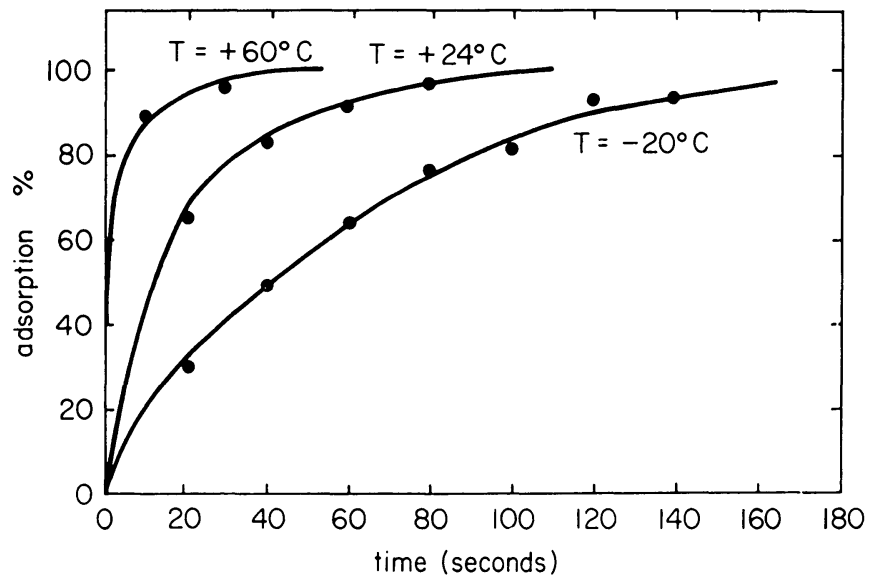

Fig. 3. Adsorption of $\mathrm{O}_{2}$ by white phosphorus, applied to pumice at different temperatures.

oxygen adsorption curves for white phosphorus deposited on pumice are shown in Figure 3. However, the phosphorus adsorption reaction has a strong dependence on temperature and at low temperatures proceeds with insufficient speed. This circumstance forced us to investigate the possibility of heating the experimental apparatus.

As an adsorber we used red phosphorus, which upon sublimation changes over to the white modification, energetically adsorbing oxygen. This method insures both the inertness of the reagent during the time of its storage and the independence of the speed of the reaction on the temperature of the surrounding medium. To insure the mechanical strength of the adsorber, a binder was used (a solution of potassium bichromate and sodium silicate).

This method was used only in the first experiment on Venera-4 when the possibility of a large oxygen content in the atmosphere of the planet had not been excluded. 
However, Venera-4 showed that the oxygen content in the atmosphere of Venus is small. Because of this the following spacecraft used only the thermochemical method, intended for threshold detection of small quantities of oxygen.

In view of the great chemical activity of oxygen, one can select a whole series of solids that react with oxygen when heated.

The refractory metals are of greatest interest, since there is a great difference among their temperatures of oxidation and fusion. For the determination of oxygen they can be used in the form of a fine wire (a filament or coil). The resistance of the heated wire increases on reaction with oxygen as a result of an increase in temperature caused by a noticeable thermal effect of the reaction, and as a result of a decrease in the wire's cross-section on being oxidized.

The use of a refractory wire in itself makes its heating easy. The most suitable metal was found to be tungsten. Its reaction with oxygen in the interval $700-1000{ }^{\circ} \mathrm{C}$ has a sufficiently distinctive character. In pure nitrogen and in carbon dioxide, as our experiments showed, burn-out of tungsten coils does not occur in the course of extended periods of time. The addition of a small amount of oxygen (partial pressure of a few torr) leads to the functioning of the detector in the required length of time. For oxygen determination we used a coil of tungsten wire having diameter $12 \mu$.

The results of the measurements of the oxygen content of the atmosphere of Venus are given in Table III. As can be seen from the table, oxygen was measured seven times. All measurements (except one) indicate the possibility of only a very small amount of oxygen in the atmosphere of Venus. One measurement (greater than $0.4 \%$ ) obtained on Venera- 4 could be connected with a less precise determination than the other measurements made on Venera-5 and Venera- 6.

TABLE III

Results of the determination of $\mathrm{O}_{2}$ in the atmosphere of Venus

\begin{tabular}{llcll}
\hline Number & $\begin{array}{l}\text { Pressure } \\
\text { (atm) }\end{array}$ & $\begin{array}{l}\text { Temperature of } \\
\text { the atmosphere } \\
\text { of Venus, }{ }^{\circ} \mathrm{C}\end{array}$ & $\begin{array}{l}\text { Results of } \\
\text { measurements } \\
\text { in Volume } \%\end{array}$ & Spacecraft \\
\hline 1 & 0.7 & $\sim 25$ & $>0.4$ & Venera-4 \\
2 & 2 & 85 & $<1.5^{\mathrm{a}}$ & Venera-4 \\
3 & 2 & 85 & $<0.3$ & Venera-6 \\
4 & 5 & 150 & $>0.1$ & Venera-5 \\
5 & 5 & 150 & $<0.1$ & Venera-5 \\
6 & 10 & 220 & $<0.1$ & Venera-6 \\
7 & 10 & 220 & $<0.2$ & Venera-6 \\
\hline
\end{tabular}

a Data obtained by the manometric method. All others by thermochemical means.

Thus the content of oxygen in the atmosphere of Venus does not exceed $0.1 \%$. This content is the limit of sensitivity of our instruments. Because of this it was not possible to carry out determinations of still smaller quantities of $\mathrm{O}_{2}$. The limit given here does not contradict data on the content of various forms of oxygen in the ionosphere of Venus. 
D. THE DETERMINATION OF MOISTURE

Two methods were used in the determination of moisture; the manometric method for large quantities, and the electrolytic method for small quantities.

In order to choose the adsorbant for use in the manometric method, comparisons were made of the effectiveness of various substances such as $\mathrm{P}_{2} \mathrm{O}_{5}$ (phosphorus anhydride), $\mathrm{KOH}, \mathrm{CaCl}_{2}$, and others. In the present instruments the desiccant $\mathrm{CaCl}_{2}$ was used. Its desiccating properties have been well studied. The advantage of $\mathrm{CaCl}_{2}$ lies in its inertness relative to other gaseous components and their adsorbants. The pure calcium chloride used was first fused, ground up, and then sifted without exposure to moisture. In use, the sifted fraction with grain sizes 1-3 mm achieved practically complete adsorption of moisture in the instrument in 30-60 sec. (Figure 4).

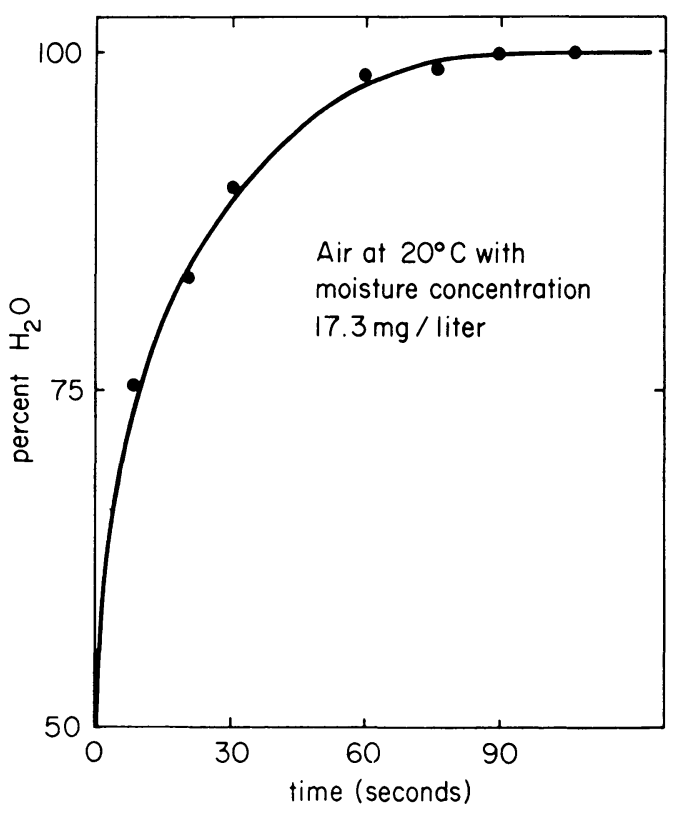

Fig. 4. . Adsorption of $\mathrm{H}_{2} \mathrm{O}$ by calcium chloride.

In the electrolytic method a sensor that operated on the principle of a change in electrical resistance of a layer of conducting substance as it adsorbs moisture was used. The moisture sensor was made in the form of a glass coil with a double winding of platinum wires in grooves on its exterior surface. As a moisture adsorbing substance to act as the conducting layer, we used hydrated phosphorus anhydride in a $20 \%$ solution of $\mathrm{P}_{2} \mathrm{O}_{5}$ in water. This was applied to the working surface of the sensor and desiccated by electrolysis. When the operating voltage is turned on, metaphosphoric acid electrolyzes to practically complete dryness, decomposing according to the reaction

$$
2 \mathrm{HPO}_{3} \rightarrow \mathrm{H}_{2}+\frac{1}{2} \mathrm{O}_{2}+\mathrm{P}_{2} \mathrm{O}_{5}
$$


In this reaction the concentration of water decreases and that of $\mathrm{P}_{2} \mathrm{O}_{5}$ increases, causing an increase in the resistance of the conducting layer and a decrease in the current. At sufficiently high concentration of $\mathrm{P}_{2} \mathrm{O}_{5}$ the layer is practically an insulator. On absorption of moisture, metaphosphoric acid is formed anew, accompanied by a fall in the resistance, thus determining the moistness of the surrounding medium.

Since $\mathrm{P}_{2} \mathrm{O}_{5}$ is a most effective adsorber of small amounts of moisture, during its long storage time the sensor was placed over an alkali to maintain its resistivity in the proper condition for effective operation. On attaining an equilibrium condition, the resistance of the coil remained within the assigned limits. The behavior of the moisture sensor under various conditions is shown in Figure 5.

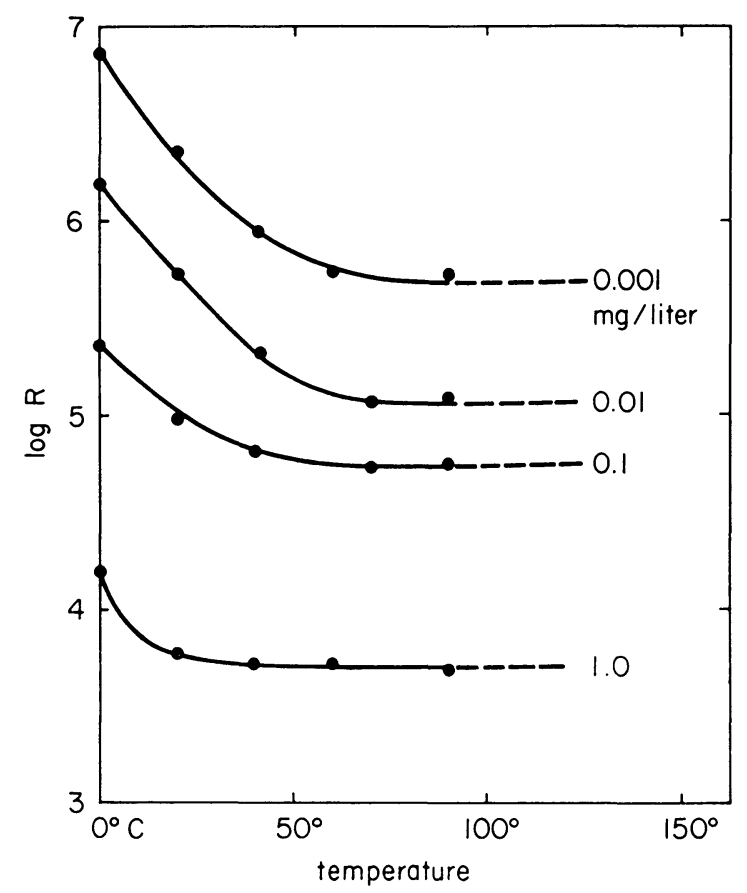

Fig. 5. Measurements of the resistance of the humidity sensor with temperature for different amounts of moisture.

The moisture sensor was situated under a glass membrane where the atmosphere entered the instrument, and during the descent of the spacecraft (after opening the instrument) the measurements of moisture were made directly in the atmosphere of the planet. The results of the measurements are presented in Table IV.

As is seen in Table IV, all the measured data attest to the existence of water in the atmosphere of Venus. Also seen is the slight tendency towards diminished water concentration with reduction of pressure. This tendency was noted from the measured humidity during the descent of the spacecraft in the atmosphere of Venus.

If we presume that the concentration of water vapor at the level of condensation is approximately $11 \mathrm{mg} / \mathrm{l}$, then the temperature of the lower part of the cloud layer must 
TABLE IV

Results of the determination of the concentration of $\mathrm{H}_{2} \mathrm{O}$ in the atmosphere of Venus

\begin{tabular}{lcclll}
\hline Number & $\begin{array}{l}\text { Pressure } \\
\text { (atm) }\end{array}$ & $\begin{array}{l}\text { Temperature of } \\
\text { the atmosphere } \\
\text { of Venus, }{ }^{\circ} \mathrm{C}\end{array}$ & $\begin{array}{l}\text { Results of } \\
\text { measurement, } \\
\text { mg/l }\end{array}$ & Adsorber & Spacecraft \\
\hline 1 & 0.6 & $\sim 25$ & $>4$ & $\mathrm{P}_{2} \mathrm{O}_{5}$ & Venera-5 \\
2 & 0.6 & $\sim 25$ & $\sim 11^{\mathrm{a}}$ & $\mathrm{CaCl}_{2}$ & Venera-5 \\
3 & 0.7 & $\sim 25$ & $>0.7$ & $\mathrm{P}_{2} \mathrm{O}_{5}$ & Venera-4 \\
4 & 2 & 85 & $<8^{\mathrm{a}}$ & $\mathrm{CaCl}_{2}$ & Venera-4 \\
5 & 2 & 85 & $\sim 6^{\mathrm{a}}$ & $\mathrm{CaCl}_{2}$ & Venera-6 \\
6 & 5 & 150 & $>0.7$ & $\mathrm{P}_{2} \mathrm{O}_{5}$ & Venera-5 \\
7 & 10 & 220 & $>0.7$ & $\mathrm{P}_{2} \mathrm{O}_{5}$ & Venera-6 \\
\hline
\end{tabular}

${ }^{a}$ These measurements were made by the manometric method, while the others were made by the electrolytic method.

be about $286 \mathrm{~K}$ (dew point). Then the atmospheric pressure at the level of condensation may be evaluated by extrapolation from the measured quantities at higher layers in the atmosphere. For the above-mentioned chemical composition of the atmosphere and the quasi-linear variation of temperature from the level of the first humidity measurement $\left(P_{1}=0.6 \mathrm{~atm}, T_{1} \simeq 25^{\circ} \mathrm{C}\right)$ to the level of condensation $\left(T_{2}=13{ }^{\circ} \mathrm{C}\right)$, the pressure at the level of condensation is about $0.5 \mathrm{~atm}$.

Using the known barometric relationship, we may also evaluate the height of the lower boundary of the cloud layer above the level of the first measurement and the temperature gradient at that point, and these correspond to $\sim 1.3 \mathrm{~km}$ and $7.5 \% \mathrm{~km}$.

Upon analysis of the atmosphere inside the cloud layer, we may make suppositions that the relationship of the pressure of water vapor to the pressure inside the cloud layer remains constant. The release of latent heat, originating upon condensation of water vapor will change the temperature gradient in accordance with the relationship

$$
\frac{\mathrm{d} T}{\mathrm{~d} h}=\left(\frac{\mathrm{d} T}{\mathrm{~d} h}\right)_{\text {adiabatic }} \cdot \frac{a+M_{\mathrm{H}_{2} \mathrm{O}}(L E / P R T)}{1+\frac{M_{\mathrm{H}_{2} \mathrm{O}}}{M} \cdot \frac{L}{P C_{p}} \cdot \frac{\mathrm{d} E}{\mathrm{~d} T}},
$$

where $a=$ the ratio of the temperature gradient under the clouds to the dry adiabatic gradient, $M_{\mathrm{H}_{2} \mathrm{O}}=$ molecular weight of water vapor, $L=$ latent heat of the phase change, $E=$ vapor tension of saturated vapor, $M=$ molecular weight of the atmosphere, $C_{p}=$ specific heat of the atmosphere at constant pressure.

On the basis of the measurements of temperature and pressure in the high layers of the atmosphere, we can determine the quantity of condensible water vapor from the formula

$$
Q=\frac{M_{\mathrm{H}_{2} \mathrm{O}}}{R T}(e-E),
$$

where $e$ is the partial pressure of water vapor in the atmosphere.

The change in humidity of the clouds with height is shown in Figure 6. From the figure it is seen that the thickness of the clouds is about $15 \mathrm{~km}$. Up to the height of 


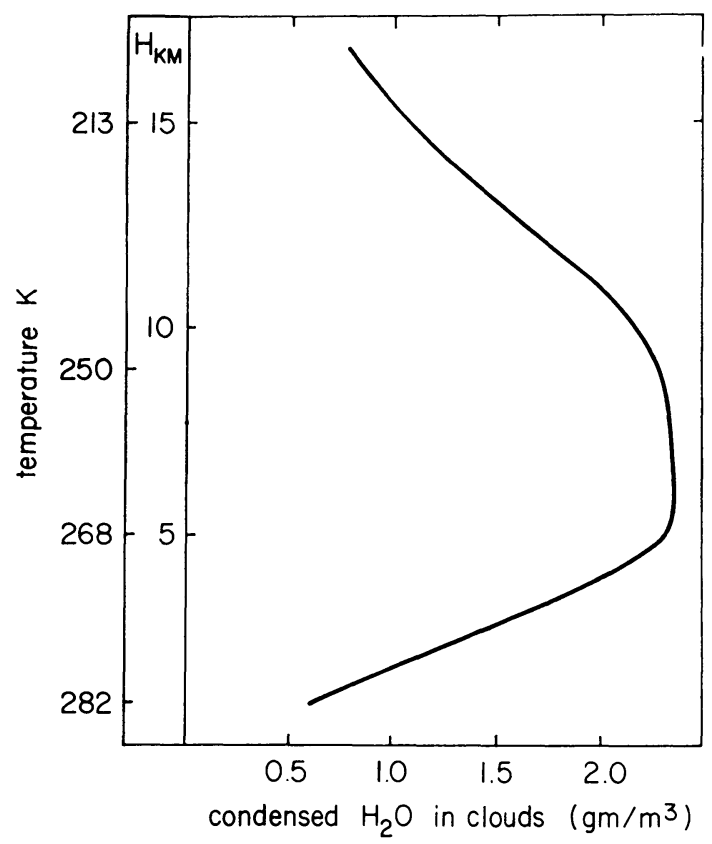

Fig. 6. Distribution of condensable $\mathrm{H}_{2} \mathrm{O}$ in the cloud layer with respect to altitude.

$7 \mathrm{~km}$ from the level of condensation, the clouds are composed, in all probability, of droplets of water, and at higher levels they consist of ice crystals. The maximum moisture content of the clouds is about $2.4 \mathrm{gm} / \mathrm{m}^{3}$ at an atmospheric temperature from $-5{ }^{\circ} \mathrm{C}$ to $-25^{\circ} \mathrm{C}$ and is situated $5-10 \mathrm{~km}$ above the level of condensation. The upper boundary of the clouds must be diffuse. Obviously, in the sub-cloud layer there exists a region of intense circulation of moisture. In this region there is falling condensation in the cloud layer. The water drops fall until they reach a region of higher temperature, are vaporized, and again moved upward. In such a case the amount of water in the atmosphere must diminish with depth.

\section{Composition and Origin of the Atmosphere of Venus}

As is known, from the Venera-4 spacecraft we determined the following composition of the Venus atmosphere:

$$
\begin{array}{ll}
\mathrm{CO}_{2} & 90 \pm 10 \% \\
\mathrm{O}_{2} & 0.4-1.5 \% \\
\mathrm{~N}_{2} & \text { No more than } 7 \% \text { (probably no more than } 2.5 \% \text { ) } \\
\mathrm{H}_{2} \mathrm{O} & 1-8 \mathrm{mg} / \mathrm{l}
\end{array}
$$

From the results of more precise and repeated determinations from the Venera- 5 and Venera-6 spacecraft as discussed above, the composition is made more exact and can be written as follows: 


$$
\begin{array}{ll}
\mathrm{CO}_{2} & 97 \pm 4 \% \\
\mathrm{O}_{2} & \text { No more than } 0.1 \% \\
\mathrm{~N}_{2} & \text { No more than } 2 \% \\
\mathrm{H}_{2} \mathrm{O} & 6-11 \mathrm{mg} / \mathrm{l} \text { (at } 0.6 \text { to } 2 \text { atm pressure) }
\end{array}
$$

Thus, the atmosphere is composed mostly of carbon dioxide gas. The concentration of nitrogen (together with the inert gases which may comprise only a small fraction of the amount of nitrogen) is not in excess of $2 \%$. The concentration of oxygen appears to be appreciably less than we found from Venera-4 and does not exceed $0.1 \%$. This quantity differs from the value determined from radioastronomical measurements of the concentration of molecular and other forms of oxygen in the ionosphere. We discussed earlier the nature of the distribution of water in the atmosphere of Venus, regarding the concentration of humidity. From all the experiments that we were able to put on the space probes, we were unable to detect in the atmosphere any other gases in any noticeable quantities. Naturally, this does not exclude the possible existence of $\mathrm{CO}, \mathrm{HF}, \mathrm{HCl}$, and $\mathrm{Ar}$ in the Venus atmosphere, and possibly other gases, but the quantities must be very small. As is known, their presence is shown in the data from ionospheric investigations.

Returning to the problem of the origin of the atmosphere of Venus [8], it may be said that the new data on the atmospheric pressure and temperature [6] show that the origin is connected with thermal effects. We had already considered the formation of large quantities of $\mathrm{CO}_{2}$ in the Venus atmosphere as following from the proximity of the planet to the sun, and the high equilibrium temperature. It seemed to us that in these conditions there occurs intensive erosion of the rocks and the composition of the atmosphere is determined in large measure by the wollastonite equilibrium

$$
\mathrm{CaSiO}_{3}+\mathrm{CO}_{2} \rightleftarrows \mathrm{CaCO}_{3}+\mathrm{SiO}_{2} \text {. }
$$

Many other reactions for the formation of wollastonite are known, for which we have experimental data on the equilibria at various temperatures and pressures. This reaction at a pressure of about 50 atm goes in the direction of the formation of wollastonite and $\mathrm{CO}_{2}$ already at temperature higher than $350{ }^{\circ} \mathrm{C}$.

We have already given an analysis of the possible concentration of $\mathrm{CO}_{2}$ on the earth and Venus. If we assume that the general reserve of $\mathrm{CO}_{2}$ on both planets is the same, then the quantity of $\mathrm{CO}_{2}$ on Venus, from other data, should be about $50 \mathrm{~atm}$. This, in general, is not in disagreement with the experimental determination of the amount of $\mathrm{CO}_{2}$, as well as the pressure and temperature we observe at the surface of Venus.

Oxygen in the atmosphere of Venus can form as a result of photodissociation of carbon dioxide gas and water. However, the low concentration of oxygen shows that it strongly recombines with the rock materials. Hydrogen, resulting from the photodissociation will disappear. It is known that Venus has a hydrogen corona, but it is not large. That is, there is a mechanism for the loss of water, or more exactly, hydrogen.

The atmosphere, as we saw, has an insignificant amount of water. The maximum $\mathrm{H}_{2} \mathrm{O}$ concentration according to our data lies in the cloud layer. One may presume 3 -P.A. 
that some quantity of water in the cloud layer occurs in the solid phase, but it requires an unambiguous determination.

The source of nitrogen-ammonia, apparently, as on the earth is degassing, a result of volcanic activity, which on Venus is very likely. Future experiments, obviously, can confirm it. We have already remarked that conditions on Venus, in comparison with those on the earth, are favourable for the rapid formation of nitrogen from ammonia.

As computations indicate, the possible share of heat that is brought up from the interior of the planet to the surface of Venus is extremely insignificant by comparison with the solar heat absorbed by the atmosphere owing to the carbon dioxide gas and water. Therefore, it is precisely this heating effect that is the main mechanism that supports and self-heats Venus and forms the heavy carbon dioxide atmosphere.

\section{References}

[1] Vinogradov, A. P., Surkov, Yu. A., Florenskii, K. P., and Andreichikov, B. M.: 1968, 'Determination of the Chemical Composition of the Atmosphere of Venus with the Interplanetary Station Venera-4', Dokl. Akad. Sci. U.S.S.R. 179, 37. (In Russian.)

[2] Vinogradov, A. P., Surkov, Yu. A., and Florenskii, K. P.: 1968, 'Chemical Composition of the Atmosphere of Venus Based on the Data of the Interplanetary Station Venera-4', $J$. Atmospheric Sci. 25, 535-536.

[3] Vinogradov, A. P., Surkov, Yu. A., and Andreichikov, B. M.: 1970, 'Investigations of the Composition of the Atmosphere of Venus with the Automatic Space Stations Venera-5 and Venera-6', Dokl. Acad. Sci. U.S.S.R. 190, 552. (In Russian.)

Moroz, V. I.: 1967, Physics of the Planets, Nauka, Moscow (English translation available in NASA Technical Translation TT F-515.

[5] Papers from the Second Arizona Conference on Planetary Atmospheres, J. Atmospheric Sci. 25, 533-671, 1968. Conference held March 11-13, 1968.

[6] 'Soviet Interplanetary Stations Venera-5 and Venera-6', Pravda 4 June, 1969. (In Russian.)

[7] Kuzmin, A. D.: 1967, Radiophysical Investigations of Venus, Levels of Science, Physics Series, VINITI Publishers, Moscow. (In Russian.)

[8] Vinogradov, A. P.: 1969, 'The Atmospheres of the Planets of the Solar System', Vestnik (Moscow State University), No. 4, Geological Series. 